\title{
SENSITIVE UNCOOLED IR DETECTORS USING GALLIUM NITRIDE RESONATORS AND SILICON NITRIDE ABSORBERS
}

\author{
Vikrant J. Gokhale ${ }^{l}$, John Roberts ${ }^{2}$ and Mina Rais-Zadeh ${ }^{l}$ \\ ${ }^{1}$ Department of Electrical Engineering and Computer Science, \\ University of Michigan, Ann Arbor, Michigan, USA \\ ${ }^{2}$ Nitronex Corporation, Durham, North Carolina, USA
}

\begin{abstract}
This paper presents the theory and measured results of a lownoise un-cooled infrared (IR) detector, which uses a combination of piezoelectric, pyroelectric, electrostrictive, and resonant effects to achieve high sensitivity. The sensor consists of a high- $Q$ gallium nitride $(\mathrm{GaN})$ micro-mechanical resonator coated with an IR absorber layer. The IR absorber converts the IR energy into heat. The generated heat causes a shift in the frequency characteristics of the $\mathrm{GaN}$ resonators. IR detection is achieved by sensing the shift in the resonance frequency of the exposed $\mathrm{GaN}$ resonator as compared to a reference resonator. A prototype GaN-based IR sensor is implemented, showing a frequency shift of $400 \mathrm{~Hz}$ per

$\mathrm{W}$ of absorbed power. The change in the beat frequency upon IR radiation is $1830 \mathrm{ppm} / \mathrm{W}$, making it possible to sense IR radiation in nano watts range.
\end{abstract}

\section{INTRODUCTION}

The detection of infrared radiation is generally accomplished using either photonic or thermal detectors, both of which have generated a large amount of research over the last two decades [1]. The relative disadvantages of thermal detectors in terms of detectivity and response time are balanced by the fact that they do not require cooling systems, reducing the system weight, power consumption, and form factor. Furthermore, thermal detectors are better suited for radiation extending into the far wavelength IR, which is extremely useful for optical astronomy. While bolometric devices have been the most popular thermal sensors commercially, there is an increasing interest in resonant IR detectors [2]-[4]. This work presents a GaN-based resonant IR sensor that utilizes the unique material properties of $\mathrm{GaN}$ to achieve high sensitivity. A reference resonator is included in the system for self-calibration (Fig. 1). Fig. 2 shows a representative scanning electron micrograph (SEM) of a GaN detector.

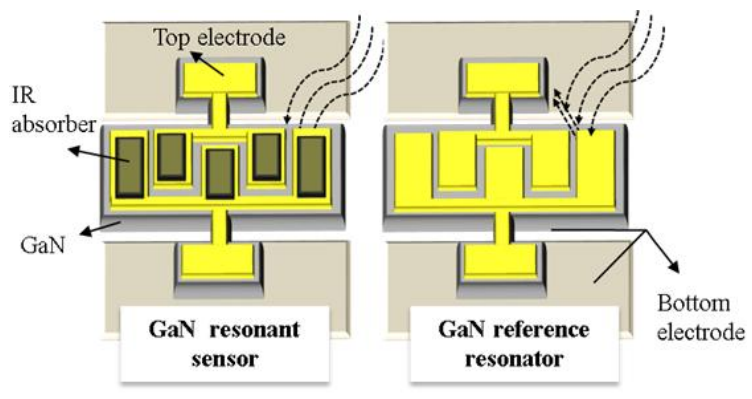

Figure 1: Schematic of a resonant sensor and a reference pair with interdigitated finger electrodes. The sensor is coated with an absorber layer. The reference resonator is either not coated with the absorber or the absorber layer on the reference resonator is blocked using the top electrode.

\section{THEORY OF OPERATION}

\section{General Resonant IR detectors}

In thermal IR detectors, radiation incident on the surface of the device increases its temperature. The conversion of incident IR

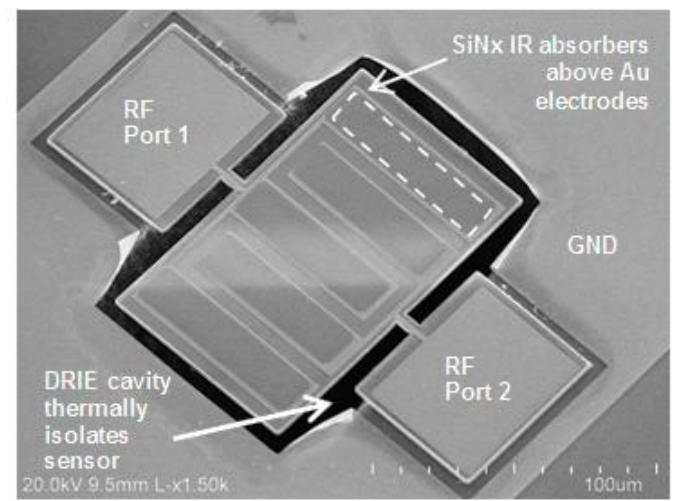

Figure 2: A SEM image of a fabricated GaN resonator with silicon nitride absorber. The dimensions of the device are 120 $\mu \mathrm{m} \times 80 \mu \mathrm{m} \times 2.15 \mu \mathrm{m}$. The tethers are $4 \mu \mathrm{m} \times 10 \mu \mathrm{m}$.

radiation power $\phi(\lambda)$ into temperature change is given by [3]

$$
\Delta T=\frac{\eta(\lambda) \phi(\lambda)}{\sqrt{G_{t h}^{2}+\omega^{2} C_{t h}^{2}}},
$$

where $G_{t h}$ is the effective thermal conductance of the device (dominated by the thermal conductance of the tethers), $C_{t h}$ is the thermal capacity of the device, $\omega$ is the rate of change of the incident signal, and $\eta(\lambda)$ is the absorption efficiency of the IR absorber layer. In resonant IR sensors, the resultant change in the resonator physical dimensions due to thermal expansion and the change in the elasticity of the resonator material are the main factors in changing the resonant frequency of the device [3]. The temperature-induced change in frequency is quantified by the wellknown temperature coefficient of frequency (TCF). Broadly speaking, the temperature dependence of the material elasticity is the dominant factor in setting the TCF and puts an upper bound on the thermal sensitivity of the resonator. By designing the device in a dually-clamped configuration and allowing it to buckle with a change in temperature, it is possible to increase the temperature sensitivity beyond TCF. However, the non-linear nature of this buckling and the fabrication challenges involved in ensuring reliable and repeatable fabrication of devices near the buckling point make this a prohibitive exercise. A second way to increase the temperature sensitivity is to use a material that has an inherently large response to heat. Pyroelectric materials are suitable candidates for this application: a pyroelectric thin film generates a spontaneous surface charge and thus a potential difference across the thickness of the open-circuit film when the temperature of the film changes (e.g., upon IR radiation). The relationship between the pyroelectric voltage and the change in temperature of a film with thickness of $t$ is given by,

$$
V=P_{V} t \Delta T
$$

Most pyroelectric materials are also piezoelectric. In a resonator made from a pyro/ piezoelectric material, this voltage $V$ can change the elastic properties of the resonator through linear 
and second-order (electrostrictive) piezoelectric interactions. In this work, we use a combination of piezo and pyroelectric effects to achieve large temperature sensitivities in resonant IR sensors.

\section{GaN Resonant IR detectors}

$\mathrm{GaN}$ is an excellent choice of material for the proposed resonant IR sensor. GaN is a single crystal wurtzite material that can be grown as a high quality thin film using metallo-organic chemical vapor deposition (MOCVD) or molecular beam epitaxy (MBE). The piezoelectric nature of $\mathrm{GaN}$ has been used to make mechanical resonators in the past by the authors [5], [6] and other researchers [7], [8]. Along with this, $\mathrm{GaN}$ is known to be a pyroelectric, with a theoretical pyroelectric voltage coefficient $\left(P_{v}\right)$ of $7 \times 10^{5} \mathrm{~V} / \mathrm{m}-\mathrm{K}$, and measured $P_{v}$ as high as $1 \times 10^{4} \mathrm{~V} / \mathrm{m}-\mathrm{K}[9]$. $\mathrm{GaN}$ is also electrostrictive [10], [11]. In $\mathrm{GaN}$, it has been found that a significant quadratic (electrostrictive) dependence of strain on electric field exists. Willatzen and Lew Yan Voon considered the case of a $\mathrm{GaN}$ thin film with $\mathrm{AC}$ and $\mathrm{DC}$ fields superimposed and derived the effect of the electrostriction on the acoustic velocity $v$ of the material [11]. Following their analysis for the acoustic velocity along the resonator length, we get,

$$
v=\sqrt{\frac{\epsilon_{31}^{\prime} c_{11}+e_{31} e_{31}^{\prime}}{\rho\left(\epsilon_{31}^{\prime}+2 M_{31} E_{3} e_{31}^{\prime}\right)}},
$$

where $\epsilon_{31}^{\prime}=\left(\epsilon_{31}-M_{31} E_{3} e_{31}\right), e_{31}^{\prime}=\left(e_{31}+M_{31} E_{3} c_{31}\right)$, and the coefficients $\epsilon, e$ and their corresponding primed counterparts represent permittivity and piezoelectric strain tensors, respectively. $M_{31}$ is the electrostrictive transverse coefficient and $E_{3}$ is the electric field across the thickness of the film. The change in the acoustic velocity is directly related to the resonant frequency. For a length-extensional resonator we have

$$
f=\frac{n}{2 L} \sqrt{\frac{C_{e f f}}{\rho}}=\frac{n v}{2 L},
$$

where $C_{e f f}$ is the effective stiffness, $\rho$ is the mass density, and $n$ is the mode of operation. Based on published values for the material properties of $\mathrm{GaN}$ and a $M_{31}$ value of $-6 \times 10^{-19} \mathrm{~m}^{2} \mathrm{~V}^{-2}$, we get the relative frequency sensitivity to voltage as large as -1000 ppm/V (Fig. 3).

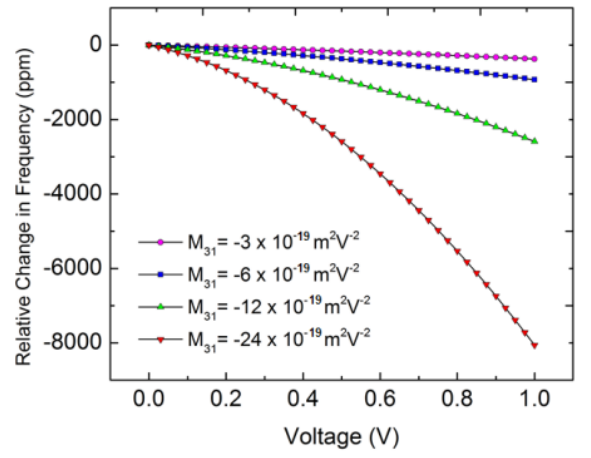

Figure 3: Predicted values of resonant frequency change due to electrostriction. For $M_{31}=-6 \times 10^{-19} \mathrm{~m}^{2} / V^{2}$ (half of $M_{33}$ ) the relative frequency change is $-1000 \mathrm{ppm} / V$ (The accurate value of $M_{31}$ is not known). The plot is derived based on the theoretical model for electrostriction in GaN [11].

The pyroelectric-electrostrictive interaction of $\mathrm{GaN}$ can be utilized as the detection mechanism for implementing resonant IR detectors. For GaN resonators presented in this work, with a thickness of $2.15 \mathrm{~m}$, and assuming a conservative value of $1 \times 10^{5}$ $\mathrm{V} / \mathrm{m}-\mathrm{K}$ for $P_{v}$, a temperature induced frequency shift of -215 $\mathrm{ppm} / \mathrm{K}$ can be achieved, which is an order of magnitude greater than the measured TCF for $\mathrm{GaN}$ resonators (i.e., $-17.7 \mathrm{ppm} / \mathrm{K}$ [5]). Thus, along with the TCF, the electrostrictive perturbation in GaN can be explored to enhance the sensitivity of thermal detectors.

\section{Sensor Scheme}

The IR sensors must be designed to work reliably at conditions that are sub-optimal for performance, in the presence of interfering signals due to shock, acceleration, and changes in ambient temperature. The best way to cancel out these interfering effects is to use a reference resonator that is similar to the sensor in all ways except one: the surface of the reference resonator does not have the absorber layer exposed. The small amount of density difference caused by the absorber and the expected variations in fabrication offset the frequency of the reference by a small amount, as compared to the sensor. This difference is enough to cause a small beat frequency which can be used as a reference to compare the frequency shift of the sensor with. In order to reduce this beat frequency, further design iterations of the reference can have the absorber layer, but coated with half the thickness of the top electrode. The frequencies of the two resonators can be described as functions of temperature by [12]

$$
\begin{aligned}
& f_{s}\left(T_{s}\right)=f_{s}\left(T_{0}\right)+A_{s} \Delta T_{s}+B_{s} \Delta T_{s}{ }^{2}+\cdots \\
& f_{r}\left(T_{r}\right)=f_{r}\left(T_{0}\right)+A_{r} \Delta T_{r}+B_{r} \Delta T_{r}{ }^{2}+\cdots
\end{aligned}
$$

Neglecting non-linear temperature dependence, we can write the beat frequency as

$$
f_{B}(T)=f_{s}\left(T_{s}\right)-f_{r}\left(T_{r}\right)=\left[A_{s} \Delta T_{s}-A_{r} \Delta T_{r}\right]+f_{B}\left(T_{0}\right)
$$

The subscripts $s, r$, and $B$ indicate the sensor, reference, and beat frequencies, respectively. $T_{0}$ is the initial temperature. The coefficients $A_{i}$ are the net coefficients of frequency change with respect to temperature, inclusive of all contributing mechanisms. Thus, the relative change in the beat frequency is a function of the incident IR radiation (Eq. 8). The beat frequency and the change thereof are easily measured using a mixer [12].

IR sensitivity $=\frac{\Delta f_{B} / f_{B}\left(T_{0}\right)}{\phi(\lambda)}=\frac{\left[A_{S} \Delta T_{S}-A_{r} \Delta T_{r}\right] / f_{B}\left(T_{0}\right)}{\phi(\lambda)} \approx \frac{A_{s} \eta(\lambda)}{G_{t h} f_{B}\left(T_{0}\right)}$

\section{EXPERIMENTAL RESULTS \\ Measurement setup}

The experimental setup for measuring the frequency response of the GaN resonators is shown in Fig. 4.

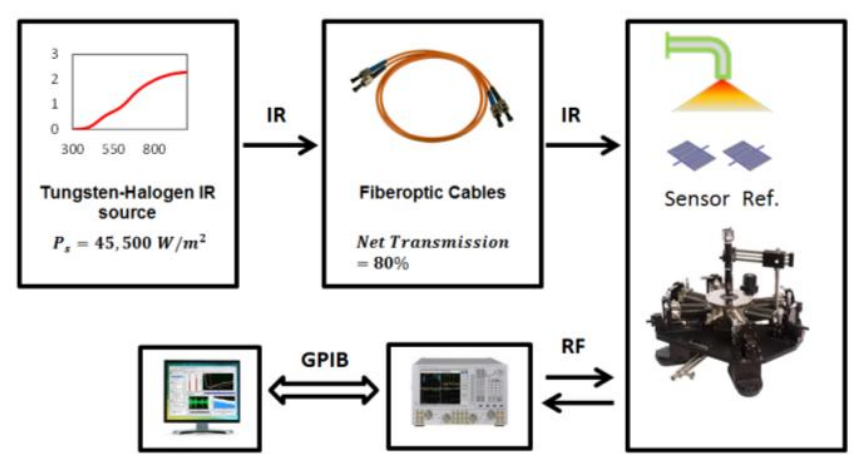

Figure 4: Schematic showing the IR measurement setup of the GaN resonators. Both sensor and reference resonators are measured using an identical procedure. 
The GaN sensor is placed in a temperature controlled probe station. IR energy is provided by a Tungsten-Halogen lamp and is coupled into the probe station using optical fibers. The RF performance of the GaN resonators is measured using an Agilent Vector Network Analyzer.

\section{Absorber Efficiency}

As seen in Equation (8), a significant factor in setting the sensitivity is the absorptivity $\eta(\lambda)$ of the IR absorber layer. GaN is only selectively absorbing at UV wavelengths. Furthermore, the metal electrodes needed for piezoelectric actuation would have highly reflecting surfaces. For example gold is almost perfectly reflecting at any thickness above $17 \mathrm{~nm}$ [13]. One solution is to coat the electrodes with a material with higher absorptivity. In this work, the GaN resonators are coated with low temperature PECVD silicon nitride $\left(\mathrm{SiN}_{\mathrm{x}}\right)$. Silicon nitride has an absorption efficiency of about 0.1 to 0.2 in the near IR spectrum (Fig. 5). To improve sensitivity, the absorber layer can be made out of carbon nanotubebased nanocomposites with an absorption coefficient of more than 0.9 over a broad spectrum [14].
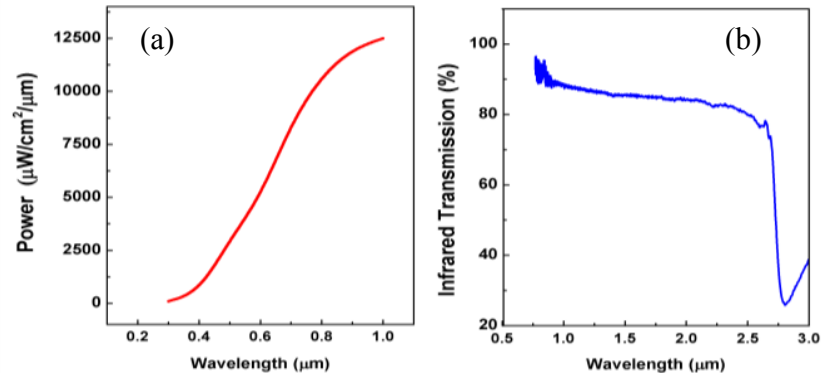

Figure 5: (a) Spectral distribution of the power supplied by the IR source. (b) Transmission spectrum for a $50 \mathrm{~nm}$ thick $S_{i} N_{x}$ film. In the relevant spectral range, $<1 \mu \mathrm{m}$, the film absorbs 10 to $20 \%$ of incident IR power. More efficient broadband absorbers can further improve the device sensitivity.

The nominal RF response of the resonator and the subsequent change when it is subject to IR radiation is given in Fig. 6. Based on the power output spectrum of the IR source, and taking into consideration fiber losses, we can calculate the power absorbed by the sensor to be 56 W. Fig 7 shows the difference in the frequency response of this sensor and a reference resonator (without the coating). From Fig. 7, the relative shift in beat frequency is $22 \mathrm{kHz}$, which gives a sensitivity of $\sim 1830 \mathrm{ppm} / \mathrm{W}$. The change in the reference frequency is only $\sim 2 \mathrm{kHz}$, uncorrelated to the IR signal, and due to random fluctuations. This gives us a minimum detectable power of $\sim 9 \mathrm{nW}$.

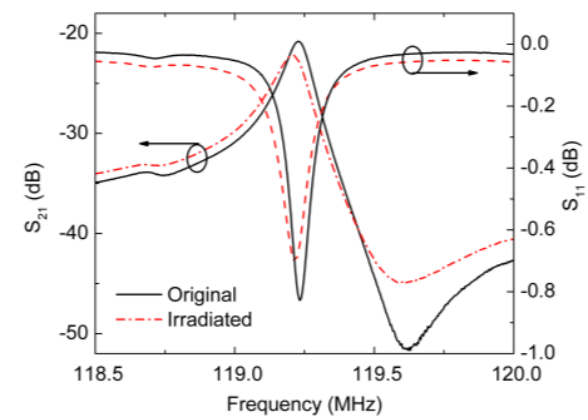

Figure 6: The change in the frequency response of a $160 \mu \mathrm{m} \times 80$ $\mu \mathrm{m} \times 2.15 \mu \mathrm{m}$ GaN resonator operated at its $5^{\text {th }}$ length-extensional mode upon radiation with $\sim 56 \mu \mathrm{W}$ IR power.
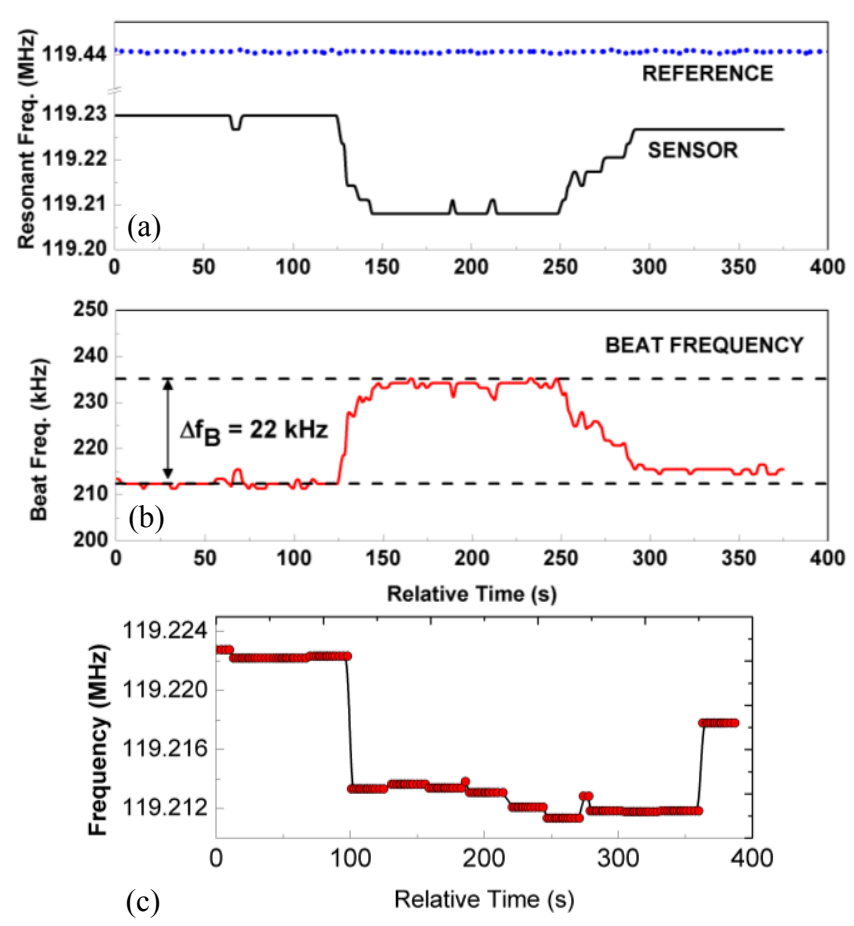

Figure 7: (a) The change in frequency of the sensor and a reference resonator on application of the IR signal. The reference resonator is identical to the sensor, except it does not have the SiNx coating. Beat frequency between the two exhibits a large change of $-21.8 \mathrm{kHz}(-183 \mathrm{ppm})$. The change in the beat frequency upon IR radiation is $10 \%$ of the original value. This can be readily detected using a mixer. (c) Faster scans reduce the overhead time due to the measurement system, but do not reduce parasitics.

The slow response seen in Fig. 7 is not the characteristic of the device, but rather of the thermal parasitics due to the probes, measurement speed, and delays inherent in the data acquisition system. We can verify this by acquiring faster (but less sensitive) scans (Fig. 7 (c)).
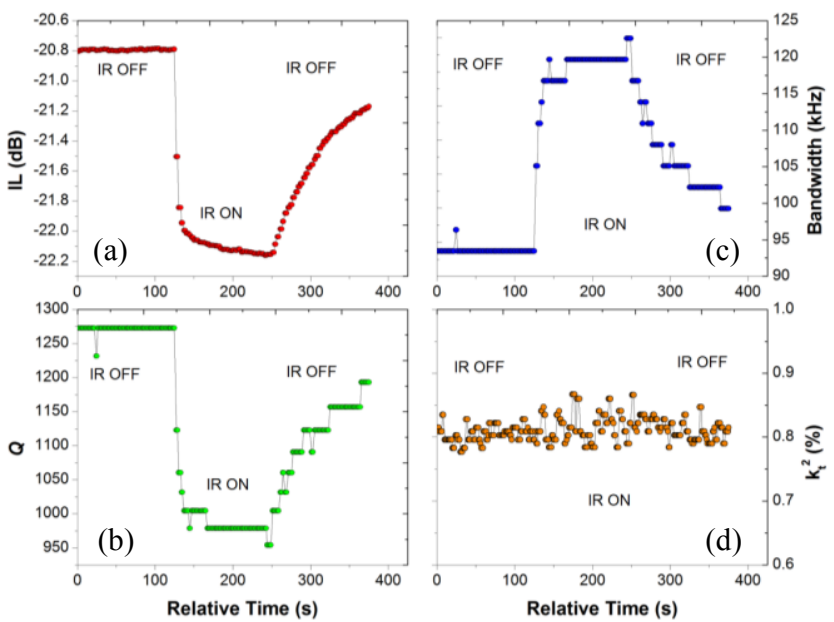

Figure 8: Figure 2: (a) Insertion loss, (b) Q, (c) bandwidth and (d) effective coupling coefficient $\left(k_{t}^{2}\right)$ of the resonator as functions of the incident IR signal. These quantities can be used in alternate measurement schemes instead of frequency sensing.

Apart from the frequency, the resonator undergoes various other changes in its performance (Fig. 8) [15], [16]. The $Q$ and the 
loss both degrade, and the bandwidth increases due to the addition of the thermal energy to the resonant system. Changes in these parameters can be used in different types of sensing schemes. Table 1 shows relevant shifts in resonator parameters for three measured sensors. The measurement conditions are the same for all devices. The important metrics for comparison of thermal detectors are the detectivity $\left(\mathbf{D}_{\mathrm{th}}^{*}\right)$, response time, and thermal fluctuation noise floor (the dominant noise mechanism for mechanical resonators [3], [17]). For the range of resonator sizes in this work, the thermal noise floor is in the range of $1 \mathrm{~K}$ to $10 \mathrm{~K}$ at room temperature. Using this design, it is possible to achieve detectivity on the order of $1 \times 10^{9} \mathrm{~cm} . \mathrm{Hz}^{0.5} . \mathrm{W}^{-1}$, with response time on the order of 1 ms (Fig. 9 (a)) [15]. Using better absorber layers, it is also possible to improve the sensitivity of the system (Fig. 9 (b)).

Table 1: Measured results for three different GaN IR sensors.

\begin{tabular}{|c|c|c|c|}
\hline & $\begin{array}{c}\text { Sensor 1 } \\
\text { (Fig. 6) }\end{array}$ & Sensor 2 & Sensor 3 \\
\hline \hline Dimensions & $\begin{array}{c}\mathbf{1 6 0} \boldsymbol{\mu \mathbf { m } \times} \\
\mathbf{8 0} \boldsymbol{\mu \mathbf { m }}\end{array}$ & $\begin{array}{c}\mathbf{1 6 0} \boldsymbol{\mu \mathbf { m } \times} \\
\mathbf{8 0} \boldsymbol{\mu \mathbf { m }}\end{array}$ & $\begin{array}{c}\mathbf{1 2 0} \boldsymbol{\mu m} \times \\
\mathbf{8 0} \boldsymbol{\mu m}\end{array}$ \\
\hline \hline Original f $\mathbf{~ ( M H z ) ~}$ & 119.2267 & 119.4547 & 191.6475 \\
\hline$\Delta \mathbf{f}(\mathbf{k H z})$ & -21.861 & -15.615 & -10 \\
\hline \hline Original $\boldsymbol{Q}$ & 1272.566 & 813.83 & 2323 \\
\hline$\Delta \boldsymbol{Q}$ & -318.33 & -216.289 & -406.63 \\
\hline \hline Original IL (dB) & -20.804 & -24.023 & -16.522 \\
\hline$\Delta \mathbf{I L}(\mathbf{d B})$ & -1.375 & -1.309 & -1.487 \\
\hline \hline
\end{tabular}
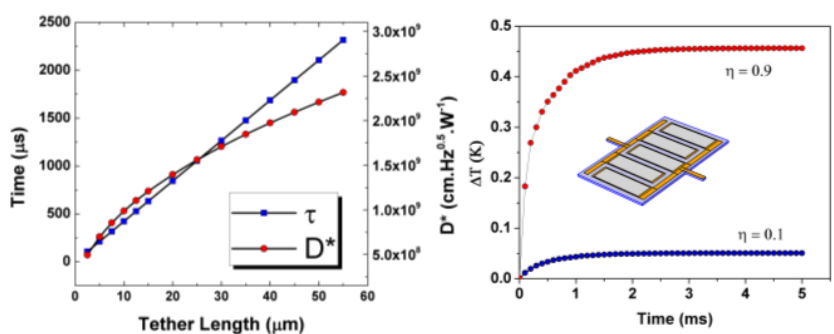

Figure 9: (a) $D^{*}$ and time constant as a function of the tether length. For the current resonator design, with an area of $160 \mu \mathrm{m} \times$ $80 \mu \mathrm{m}$, tether length of $20 \mu \mathrm{m}$ gives an optimal value for these parameters. (b) The response time of $1 \mathrm{~ms}$ is verified using COMSOL simulations for this model (inset). Improving the absorber efficiency from 0.1 to 0.9 will improve the thermal sensitivity.

\section{CONCLUSION}

Resonant IR sensors made from GaN with a sensitivity of $1830 \mathrm{ppm} / \mathrm{W}$ has been demonstrated using a sensor-reference pair. Utilizing a reference resonator enables large, perceptible changes in beat frequency due to irradiation, while cancelling out the effects of shock, acceleration, and ambient thermal change. A practical system would need further design optimization to get the best NEDT, D* and response time. The use of a broadband absorber with improved efficiency will improve the sensitivity of the detector by at least five times. Integration of the absorber and complete integration of the sensor-reference pair is ongoing work.

The interface circuitry required to measure frequency shifts can be co-fabricated on the same substrate, as $\mathrm{GaN}$ is a good material for high speed transistors. Alternate measurements schemes are also possible, using the same device. The use of the resonant detectors presented in this work is the first step to realizing IR focal plane arrays based on resonant sensors.

\section{ACKNOWLEDGENTS}

This work has been funded by NSF under Grant \#1002036, and MAST CTA under contract W911NF. The authors thank the staff at the Lurie Nanofabrication Facility for fabrication support. Travel support has been generously provided by the Transducer Research Foundation.

\section{REFERENCES}

[1] A. Rogalski, "Infrared detectors: status and trends," Progress in Quantum Electronics, 27, pp. 59-210, 2003.

[2] C. Cabuz et al., "Fabrication and packaging of a resonant infrared sensor integrated in silicon," Sensors and Actuators A, 43, pp. 92-99, 1994.

[3] J. R. Vig, R. L. Filler, and Y. Kim, "Uncooled IR imaging array based on quartz microresonators," J. Microelectromech. Sys., vol. 5, pp.131-138, 1996.

[4] P. Kao, and S. Tadigadapa, "Micromachined quartz resonator based infrared detector array," Sensors and Actuators A, vol. 149, pp. 189-192, 2009.

[5] V. J. Gokhale, J. Roberts, and M. Rais-Zadeh, "High performance bulk mode gallium nitride resonators and filters," Transducers, Beijing, PRC, pp. 926-929, 2011.

[6] A. Ansari et al., "Gallium nitride-on-silicon micromechanical overtone resonators and filters," IEEE Electron Devices Meeting, pp. 485-488, 2011.

[7] M. Faucher et al., "Amplified piezoelectric transduction of nanoscale motion in gallium nitride electromechanical resonators," Appl. Phys. Lett., vol. 94, pp. 233506, 2009.

[8] A. Muller et al., "GaN micromachined FBAR structures for microwave applications," Superlattices and Microstructures, vol. 40, pp. 426-431, 2006.

[9] M. S. Shur, A. D. Bykhovski, and R. Gaska, "Pyroelectric and piezoelectric properties of GaN-based materials," Proc. MRS, pp. G1.6, 1998.

[10] I. L. Guy, S. Muensit, and E. M. Goldys, "Electrostriction in gallium nitride," Appl. Phys. Lett, vol. 75, pp. 3641-3643 1999.

[11] M. Willatzen and L. C. Lew Yan Voon, "Static and dynamic effects due to electrostriction in GaN/AlGaN," J. Phys.: Condens. Matt., vol. 19, pp. 506202-506221, 2007.

[12] C. M. Jha et al., "High Resolution Microresonator-based digital temperature sensor," Applied Physics Letters, vol. 91, 074101-3, August 2007.

[13] W. Lang, K. Kuhl, and H. Sandmaier, "Absorbing layers for thermal infrared detectors," Sensors and Actuators A, 34, 3, pp. 243-248, 1992.

[14] Y. Sui, V. J. Gokhale, and M. Rais-Zadeh "A thin-film infrared absorber using CNT/nanodiamond nanocomposite," Proc. MRS, April 2012.

[15] M. Rais-Zadeh, "Gallium nitride micromechanical resonators for IR detection," Invited Paper, Proc. SPIE, April 2012.

[16] V. J. Gokhale, Y. Sui, and M. Rais-Zadeh, "Novel uncooled detector based on gallium nitride micromechanical resonators," Proc. SPIE, April 2012.

[17] P. W. Kruse, "A comparison of the limits to the performance of thermal and photon detector imaging arrays," Infrared Phys. Technol., 36, pp. 869-882, 1995.

\section{CONTACT}

*V. J. Gokhale, Tel: +1-734-945-5732; vikrantg@umich.edu 